\title{
Deciphering the genetic and modular connections between coronary heart disease, idiopathic pulmonary arterial hypertension and pulmonary heart disease
}

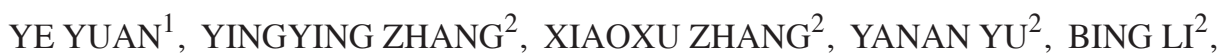 \\ PENGQIAN WANG ${ }^{2}$, HAIXIA LI $^{3}$, YIJUN ZHAO ${ }^{2}$, CHUNTI SHEN $^{1}$ and ZHONG WANG ${ }^{2}$ \\ ${ }^{1}$ Department of Respiration, Changzhou Hospital of Traditional Chinese Medicine, \\ Nanjing University of Chinese Medicine, Changzhou, Jiangsu 213003; ${ }^{2}$ Institute of Basic Research in Clinical Medicine, \\ China Academy of Chinese Medical Sciences, Beijing 100700; ${ }^{3}$ Department of Cardiology, Guang'anmen Hospital, \\ China Academy of Chinese Medical Sciences, Beijing 100053, P.R. China
}

Received June 26, 2015; Accepted April 26, 2016

DOI: $10.3892 / \mathrm{mmr} .2016 .5298$

\begin{abstract}
Coronary heart disease (CHD), idiopathic pulmonary arterial hypertension (IPAH) and pulmonary heart disease (PHD) are circulatory system diseases that may simultaneously emerge in a patient and they are often treated together in clinical practice. However, the molecular mechanisms connecting these three diseases remain unclear. In order to determine the multidimensional characteristic correlations between these three diseases based on genomic networks to aid in medical decision-making, genes from the Online Mendelian Inheritance in Man database were obtained, and applied network construction and modularized analysis were conducted. Functional enrichment analysis was conducted to explore the associations between overlapping genes, modules and pathways. A total of 29 overlapping genes and 3 common modules were identifed for the 3 diseases. Glycosphingolipid biosynthesis and the arachidonic acid metabolism are common pathways, and the biosynthetic process is suggested to be the major function involved in the three diseases. The current study reported, to the best of our knowledge for the first time, the role of glycosphingolipid biosynthesis in IPAH and PHD. The present study provided an improved understanding of the
\end{abstract}

Correspondence to: Professor Zhong Wang, Institute of Basic Research in Clinical Medicine, China Academy of Chinese Medical Sciences, 16 Nanxiaojie, Dongzhimennei, Beijing 100700, P.R. China

E-mail: zhonw@vip.sina.com

Professor Chunti Shen, Department of Respiration, Changzhou Hospital of Traditional Chinese Medicine, Nanjing University of Chinese Medicine, 25 Heping North Road, Changzhou, Jiangsu 213003, P.R. China

E-mail: czsct@163.com

Key words: co-pathogenic analysis, drug target prediction, gene interaction network, modularized analysis, network construction pathological mechanisms underlying CHD, IPAH and PHD. The overlapping genes, modules and pathways suggest novel areas for further research, and drug targets. The observations of the current study additionally suggest that drug indications can be broadened because of the presence of common targets.

\section{Introduction}

Pulmonary heart disease (PHD), idiopathic pulmonary arterial hypertension (IPAH, formerly called primary pulmonary hypertension) and coronary heart disease (CHD) are cardiovascular diseases with severe effects upon human health. CHD and PHD have a high prevalence and severe complications. The median survival time for patients with IPAH who do not receive effective drug intervention is 2.8 years, and various therapeutic strategies for IPAH are derived from treatment for pulmonary arterial hypertension $(1,2)$. Previous studies have identified in part the etiology, pathology and genetic characteristics of these conditions, which has provided guidance for prevention and treatment (3-6). However, the exact mechanisms of these diseases remain unclear, and further investigation of the biological characteristics is required in order to determine their pathological mechanisms. Previous studies have reported that the pathological processes of these diseases are not limited to the coronary or pulmonary vasculature, and that there may be systemic vasculature and complex genetic involvement during the course of these diseases $(7,8)$. Treatments with angiotensin converting enzyme inhibitors (ACEIs), vasodilators, anticoagulants, diuretics, calcium channel blockers and inotropic agents have been used to alleviate the common symptoms of PHD, IPAH, and CHD $(2,9)$, and this information enables us to explore the associations between them.

Networks can reflect the associations between genes, pathways and the diseases using network construction and modular analysis (10). A previous study based on genome-wide linkage analysis demonstrated that alstrom syndrome protein 1 is a novel genetic risk marker for early-onset myocardial infarction (a type of CHD) (11). An additional study using network 
analysis identified that patients with CHD with elevated vascular endothelial growth factor A (VEGFA) levels at baseline have an increased mortality rate compared with those with lower levels (12). Considering the complexity of the processes involved, integrated gene network analysis was used to investigate the multi-level correlation characteristics among the three diseases.

\section{Materials and methods}

Obtaining the genes and network construction. The terms 'IPAH', 'PHD' or 'CHD' were inputted into to the search box of the Online Mendelian Inheritance in Man (OMIM) database, (http://www.ncbi.nlm.nih.gov/omim), a knowledge database of human genes and genetic disorders (13). Disease-associated genes were then submitted to Agilent Literature Search software, version 2.82 (http://www.agilent. com/labs/research/litsearch.html), and an overview network of gene/protein associations was obtained.

Network analysis. Cytoscape software version 2.71 (http://www.cytoscape.org/) was used for visualization of disease-associated networks and analysis of the network properties. Network parameters including the clustering coefficient, network diameter, network centralization and network radius were determined.

Identification of modules. MCODE(version 1.32) is programme that is used for network module division (http://baderlab. org/Software/MCODE). Subsequent to the disease network data being obtained, each disease network was imported and MCODE was used to divide it into several modules using the following parameters: Connectivity threshold, 2; core threshold $\mathrm{K}, 2$; node score threshold, 0.2 .

Functional enrichment analysis. Hypergeometric distribution tests were performed to analyze the function of the modules that contained the most genes in each network (IPAH-associated, PHD-associated and CHD-associated gene networks) using the Database for Annotation, Visualization and Integrated Discovery (http://david.abcc. ncifcrf.gov/). The following parameters were used: Count, 2; EASE, 0.01; and species and background, Homo sapiens. Using the gene ontology (GO) and Kyoto Encyclopedia of Genes and Genomes annotation, the biological processes and pathway corresponding to the modules were identified, and the P-values were ranked.

\section{Results}

PHD, IPAH and CHD-associated genes in the OMIM database. Subsequent to searching the OMIM database (on May 23 , 2014), a total of 295 PHD-associated, 132 IPAH-associated and 212 CHD-associated genes were identified (Table I). A total of 29 overlapping genes were detected among the three diseases, which accounted for $9.33 \%$ (29/295) of the identified PHD-associated genes, $21.97 \%$ (29/132) of IPAH-associated genes, $13.68 \%$ (29/212) of CHD-associated genes and $5.8 \%$ $(29 / 500)$ of the total number of genes associated with the three diseases.
Topological distribution of diseases networks. Global maps of the CHD network (Fig. 1A), IPAH network (Fig. 1B) and PHD network (Fig. 1C) exhibit a similar center-outward diffuse landscape. The multiple topological parameters of the three disease networks are listed in (Table II). Based on the 295 PHD-associated genes, PHD-associated networks contained 1,363 nodes (genes) and 4,592 edges (interactions), which was more complex than the other networks. However, the IPAH-associated network had the smallest number of genes (132 IPAH-associated genes) and the biggest network centralization value (0.065) and clustering coefficient (0.658). The increase in the node degree (the number of node-edges in the network) in the CHD (Fig. 1A and D), IPAH (Fig. 1B and E) and PHD (Fig. 1C and F) networks followed a power-law distribution.

Overlapping distributions of genes in the disease networks. The 29 overlapping genes accounted for $13.68 \%$ of CHD-associated genes, $9.83 \%$ of PHD-associated genes, and $21.97 \%$ of IPAH-associated genes, 12 of which were in the 7 CHD network modules, 8 were in the 7 PHD network modules and 15 were in the 14 IPAH network modules. In addition, arachidonate 5-lipoxygenase (ALOX5) was identified in all three disease network modules.

Different overlapping distribution of modules among disease networks. The 3 overlapping modules accounted for $10.81 \%$ of the CHD modules, $2.71 \%$ of the PHD modules and $3.29 \%$ of the IPAH modules. Compared with other modules in the three diseases, $\mathrm{M}_{\mathrm{C} 919 \mathrm{P} 13}$ and $\mathrm{M}_{\mathrm{C} 19113 \mathrm{P} 25}$ ranked in a higher position.

Using the MCODE, version 1.32 for each disease network, there are 29 overlapping genes (Fig. 2A) and 3 overlapping modules in the three disease networks (Fig. 2B). Furthermore, 110 modules were identified from the CHD network (Fig. 2C), 91 modules from the IPAH network (Fig. 2D) and 74 modules from the PHD network (Fig. 2E). Notably, the three overlapping modules were identified among the three disease networks (Fig. 2B). In addition, 3, 5 and 9 additional modules, respectively, were identified between pairs of the diseases, for example $\mathrm{M}_{\mathrm{P} 1 \mathrm{C} 1}$ between PHD and CHD, $\mathrm{M}_{\mathrm{I} 3 \mathrm{P} 3}$ between IPAH and PHD and $\mathrm{M}_{\mathrm{C} 48166}$ between $\mathrm{CHD}$ and IPAH networks. Excluding the overlapping modules, the PHD network had the most unique modules $(n=93)$ compared with the other networks.

Functional enrichment analysis of three overlapping modules. Based on the GO functional enrichment analysis of the 3 overlapping functional modules in IPAH, CHD and PHD (Fig. 3A), the 3 overlapping modules were observed to contain 26 overlapping functional annotations (Fig. 3B), including 13 for biosynthetic processes (such as the cellular carbohydrate biosynthetic process and other biosynthetic processes), 8 for metabolic processes (the glycoprotein metabolic process and other metabolic processes), 4 for glycosylation processes (glycosylation, biopolymer glycosylation, protein amino acid glycosylation and protein amino acid N-linked glycosylation) and 1 for oxidation reduction. Biosynthetic processes and metabolic processes accounted for $50.00 \%$ (13/26) and 30.77\% (8/26) of all biological processes, respectively. The three overlapping modules also have two pathways (Fig. 3C), including glycosphingolipid biosynthesis and the arachidonic acid metabolism. 


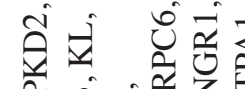

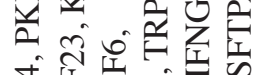

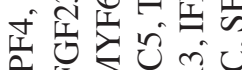

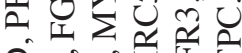

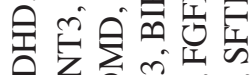

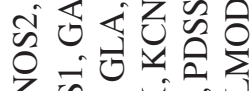
द t. 客 年的的

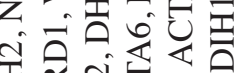
E 슬

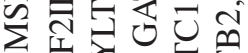
들 定

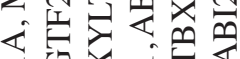

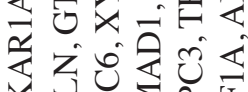

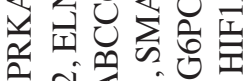

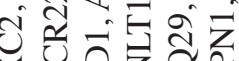

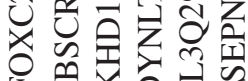

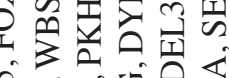
今ं के

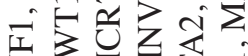

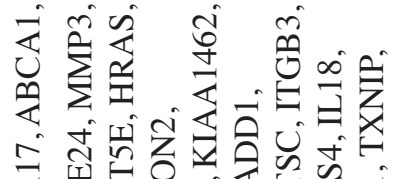

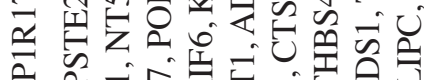

흘

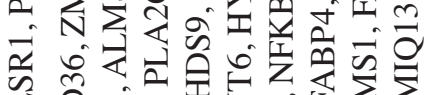

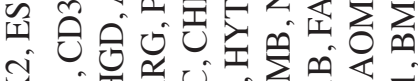

再

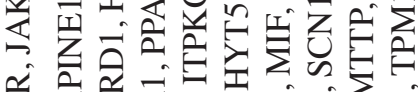

\%

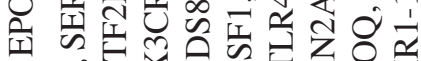

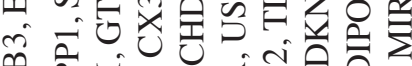

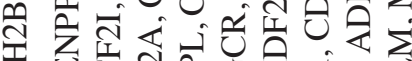
की

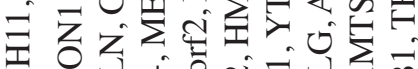

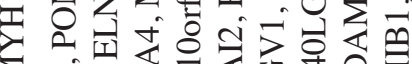
. 文讨

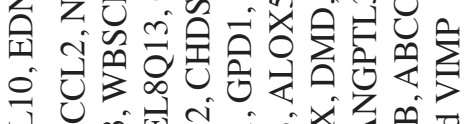

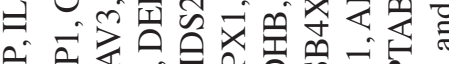

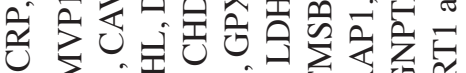

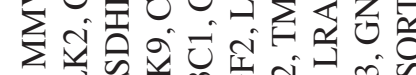

o

运

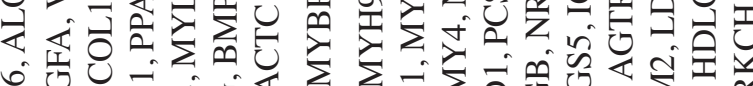
它

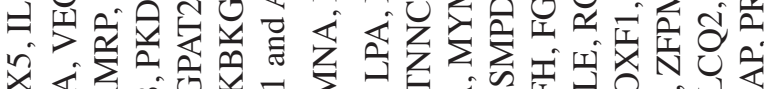

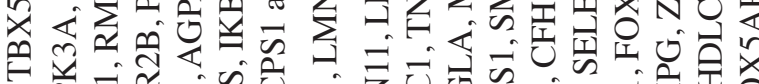
\%

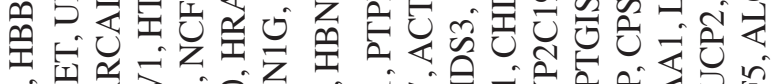

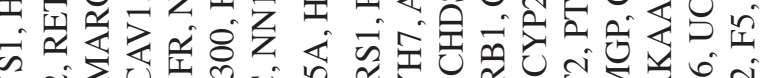
ơ 我诸

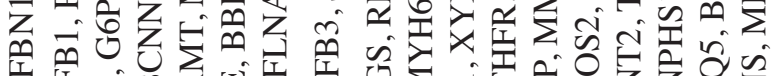

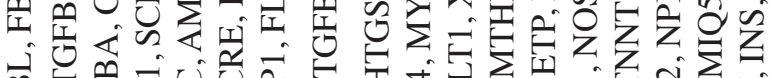
英

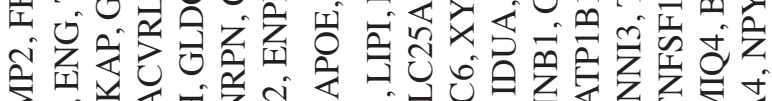

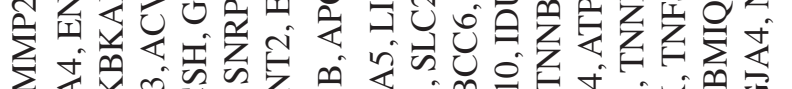

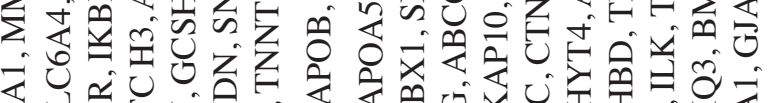

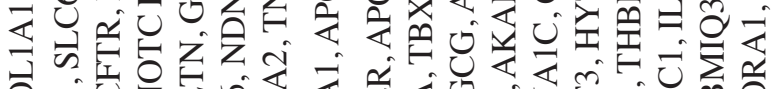
8े की

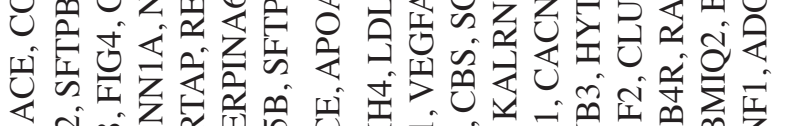
N

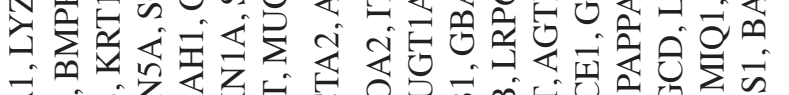

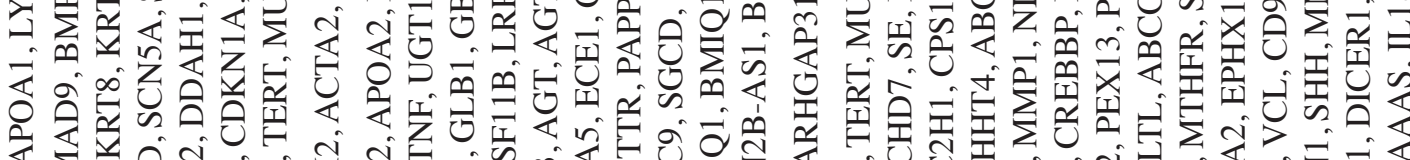

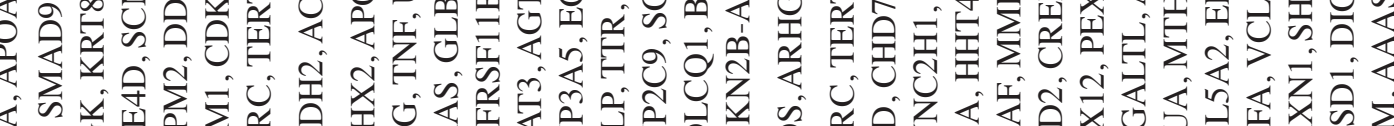

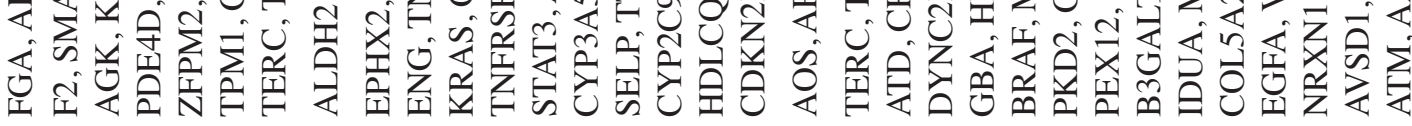

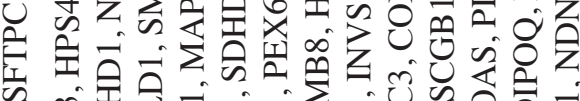

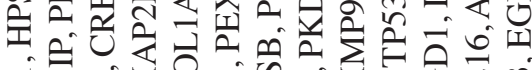

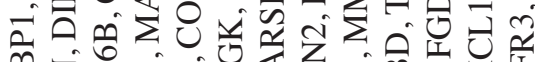

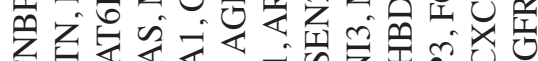

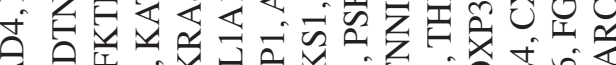

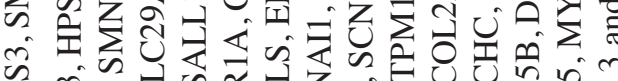
ôे nीक की

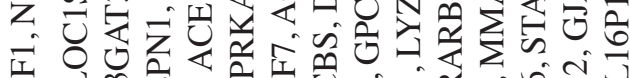

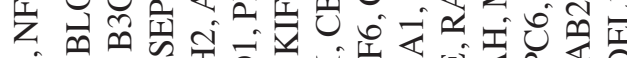
군

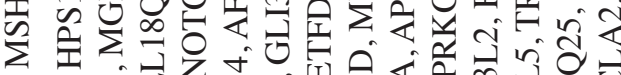

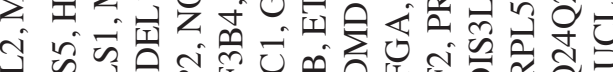

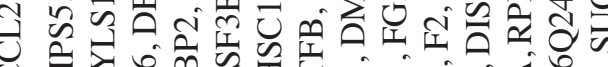

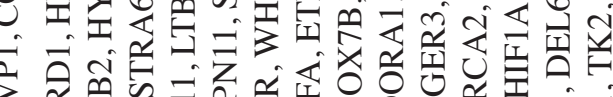

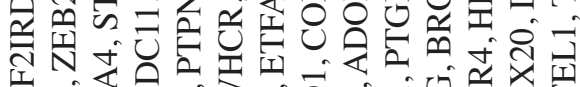

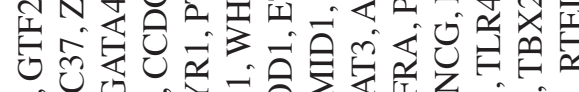

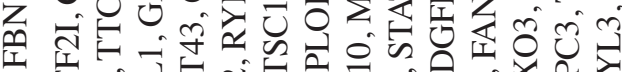

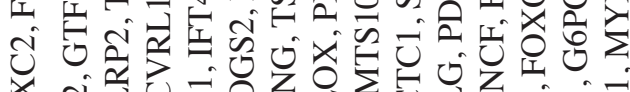
要

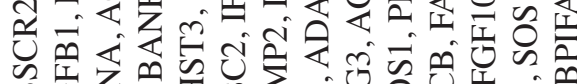

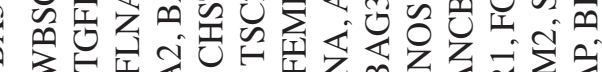

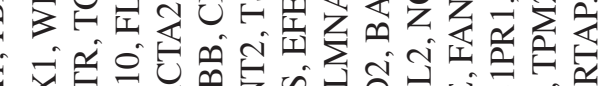

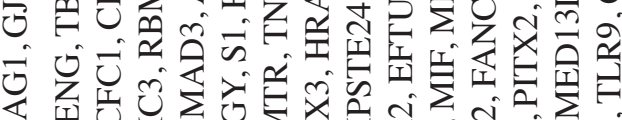

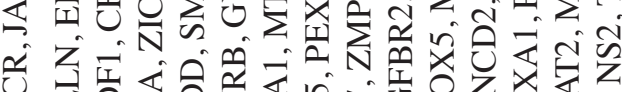

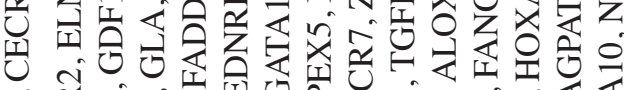
풍 어의 年

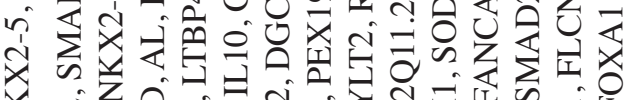

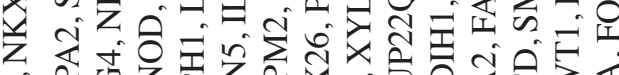

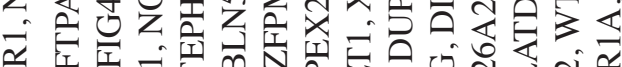

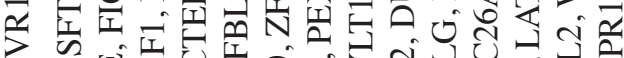
等

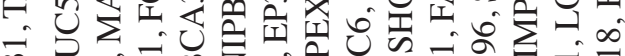

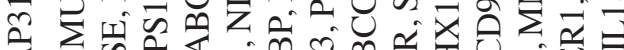
0
0
0
0
0
0
0 品 芯 $\stackrel{2}{\Xi}$

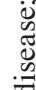
苨 $\stackrel{2}{2}$ है. 青

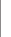

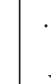

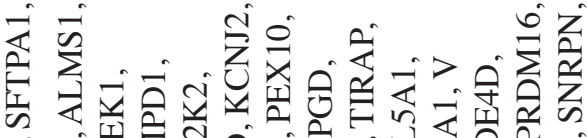

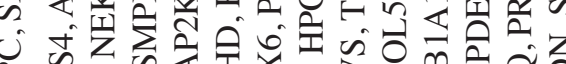

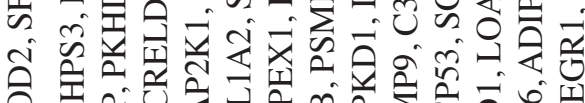

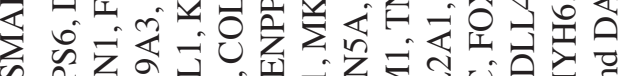

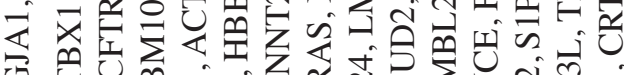


Table II. Topological parameters of the three disease networks.

\begin{tabular}{lccc}
\hline Parameter & CHD & IPAH & PHD \\
\hline Clustering coefficient & 0.611 & 0.658 & 0.614 \\
Network diameter & 13 & 13 & 12 \\
Network radius & 1 & 1 & 1 \\
Network centralization & 0.045 & 0.065 & 0.058 \\
Number of nodes & 974 & 812 & 1363 \\
Number of edges & 3040 & 2067 & 4592 \\
Gene number & 212 & 132 & 295 \\
Overlapping gene/gene & $(13.68 \%)$ & $0.21(21.97 \%)$ & $0.09(9.83 \%)$ \\
Modules & 74 & 91 & 110 \\
Average size & 7.081 & 5.374 & 6.964 \\
Maximum size & 78 & 53 & 49 \\
Minimum size & 3 & 3 & 3 \\
Modularity & 0.445 & 0.47 & 0.415 \\
Overlapping module/module & $0.04(4.05 \%)$ & $0.03(3.30 \%)$ & $0.02(2.73 \%)$
\end{tabular}

CHD, coronary heart disease; IPAH, idiopathic pulmonary arterial hypertension; PHD, pulmonary heart disease.

A

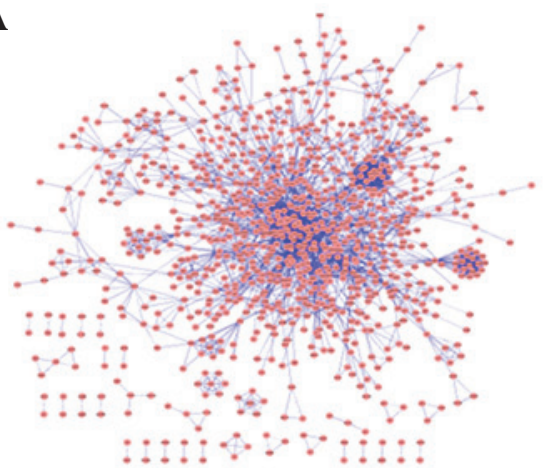

D

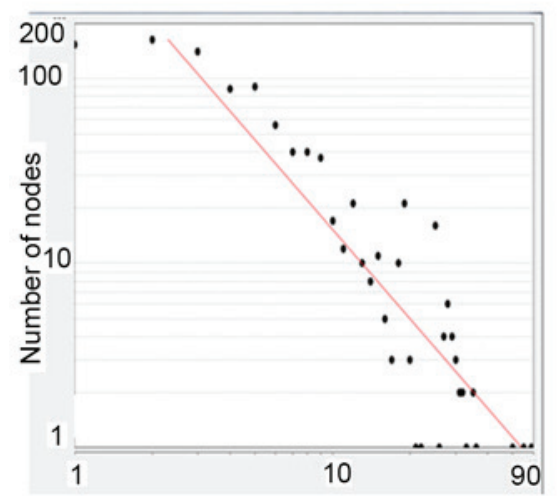

B

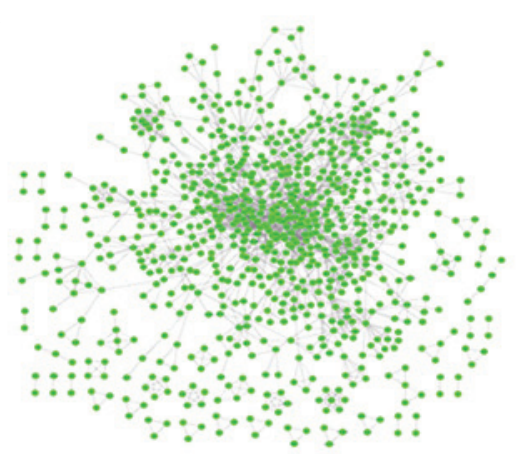

E

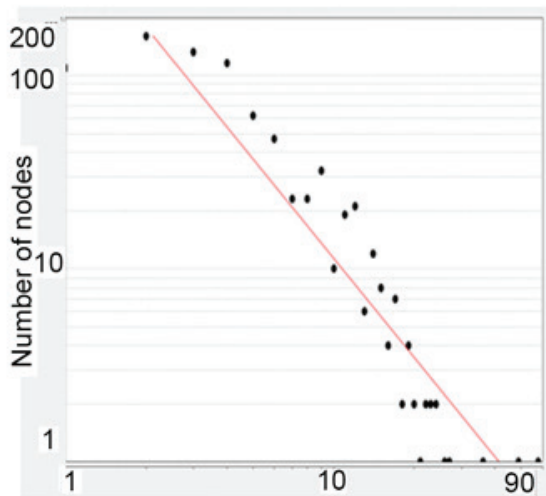

C

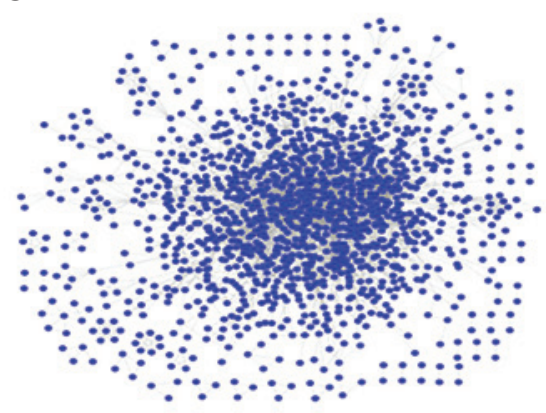

F

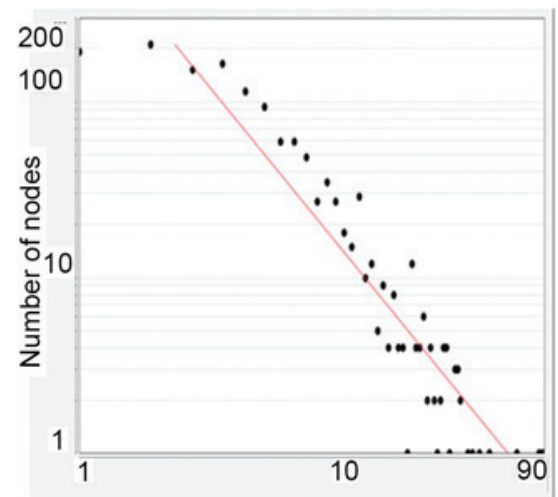

Figure 1. Characteristics of disease-associated gene interaction networks. (A) CHD network, (B) IPAH network and (C) PHD network. Node degree and power distribution of the (D) CHD, (E) IPAH and (F) PHD networks. CHD, coronary heart disease; IPAH, idiopathic pulmonary arterial hypertension; PHD, pulmonary heart disease.

Modules between IPAH and CHD. IPAH and CHD shared 3 other overlapping modules in addition to the three overlapping modules for IPAH, CHD and PHD (Fig. 3D). The 3 overlapping modules all consisted of 3 nodes and 3 edges, and had 18 functional annotations (Fig. 3E), including the eicosanoid biosynthetic process, the eicosanoid metabolic process, the fatty acid biosynthetic process and the cellular alkene metabolic process. The arachidonic acid metabolism was the only pathway identified (Fig. 3F).

Modules between CHD and PHD. In addition to the three overlapping modules, $\mathrm{CHD}$ and PHD shared 5 additional common 
A

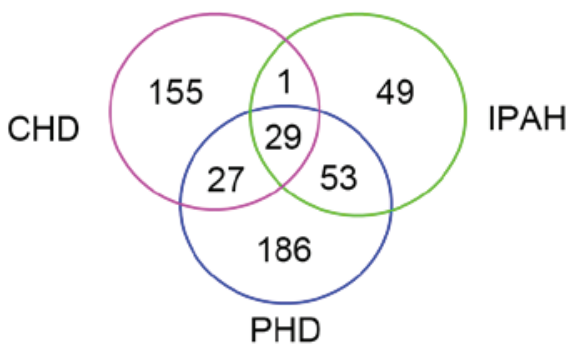

C

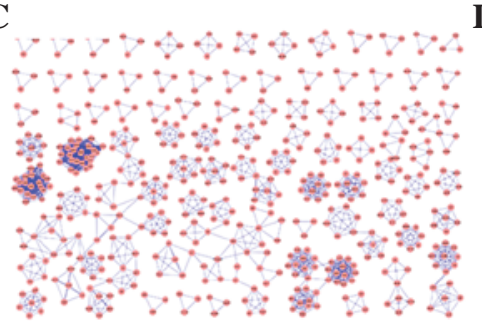

D
B

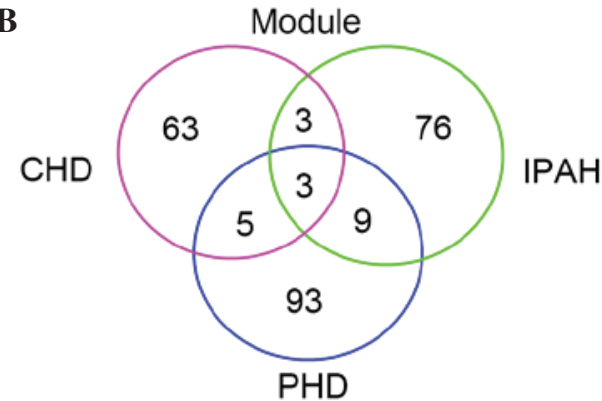

E

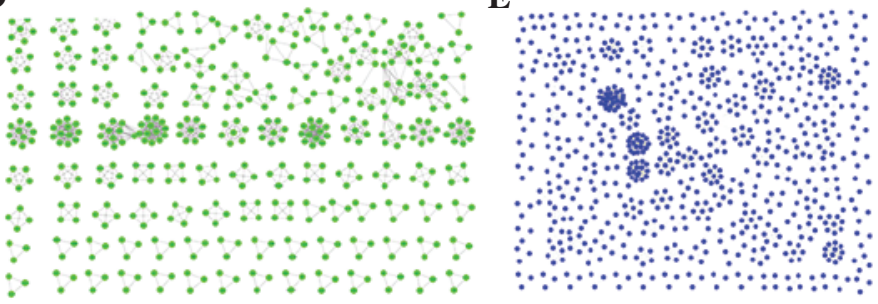

Figure 2. (A) The number of overlapping genes between CHD, IPAH and PHD. (B) The number of overlapping modules between CHD, IPAH and PHD (C) The 74 CHD modules. (D) The 91 IPAH modules. (E) The 110 PHD modules. CHD, coronary heart disease; IPAH, idiopathic pulmonary arterial hypertension; PHD, pulmonary heart disease.

A

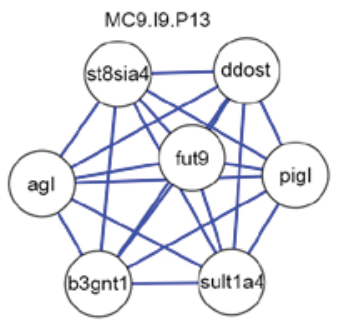

B

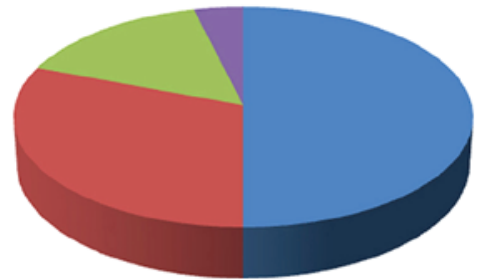

Biosynthetic process $13 \square$ Glycosylation 4

Metabolic process 8

D

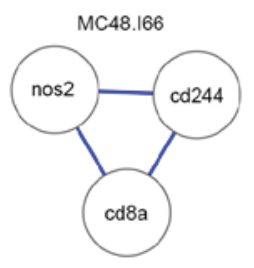

$\mathbf{E}$

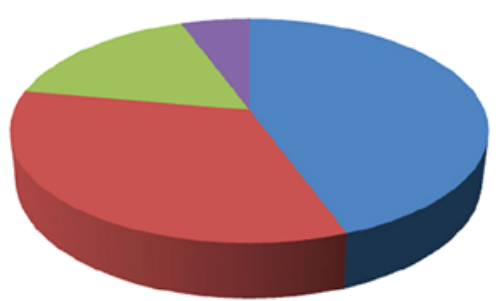

Biosynthetic process 8 Response process 3

Metabolic process 6

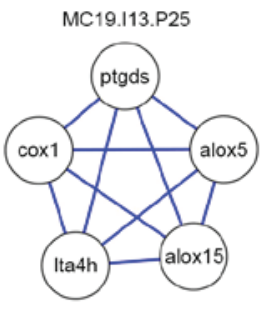

C

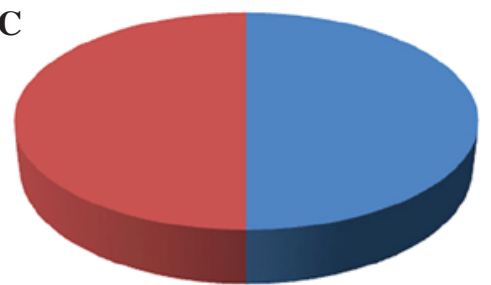

Gycosphingolipid biosynthesis

archidonic acid metabolism

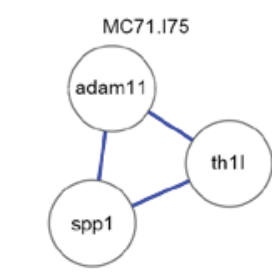

$\mathbf{F}$

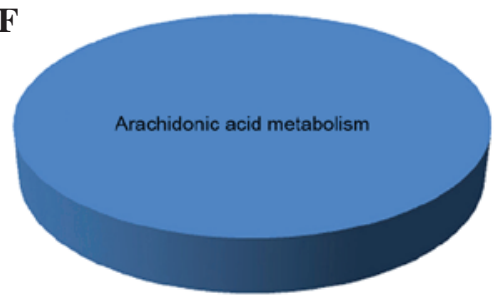

MC70.177.P102
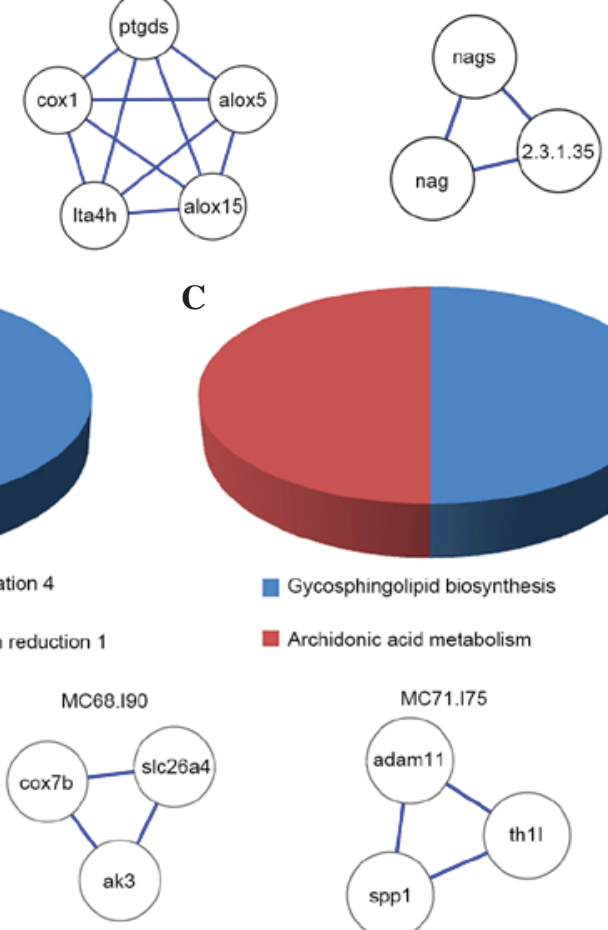


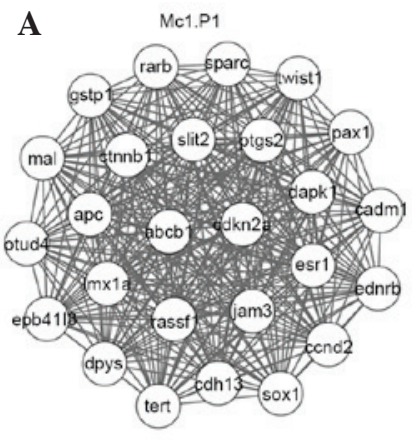

B
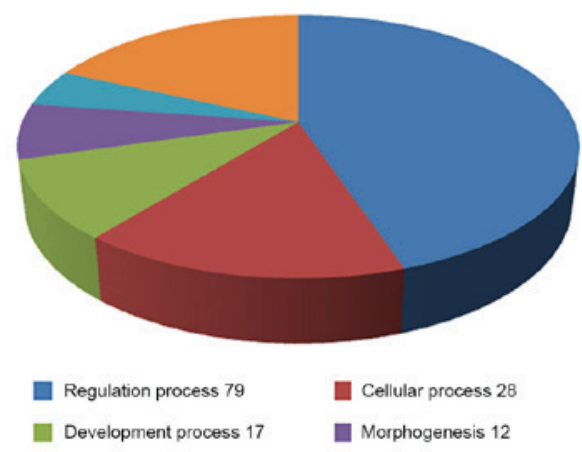

Catabolic process 8

Cellular process 28

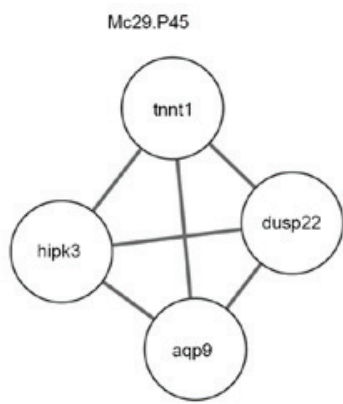

Morphogenesis 12

Others 32
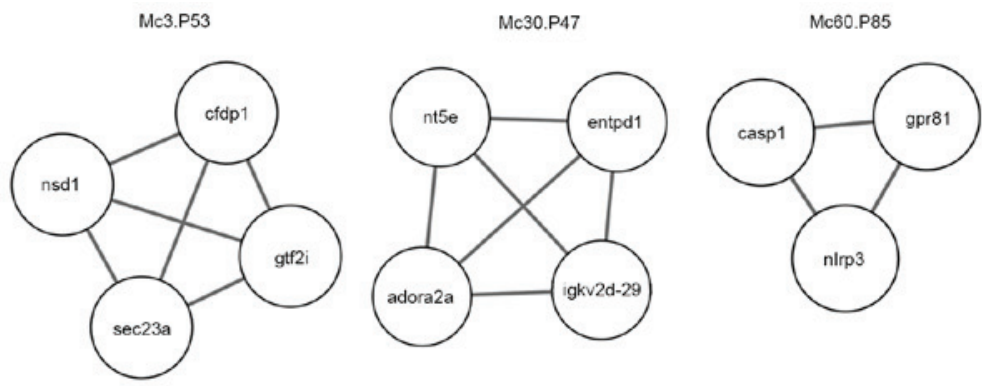

C
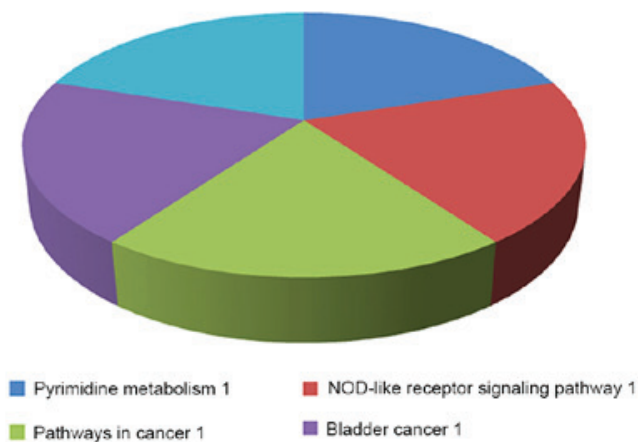

Non-small cell lung cancer 1

Figure 4. (A) The 5 overlapping modules in the CHD and PHD networks. (B) The 176 biological processes of the 5 overlapping modules in the CHD and PHD networks. (C) The 5 pathways in the 3 overlapping modules in the CHD and PHD networks. CHD, coronary heart disease; IPAH, idiopathic pulmonary arterial hypertension; PHD, pulmonary heart disease.

A

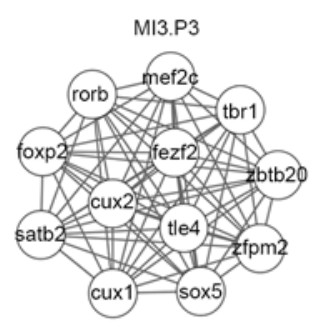

M14.P5

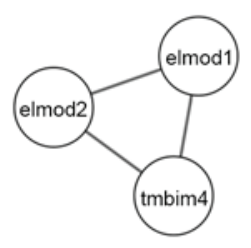

MI55.P71

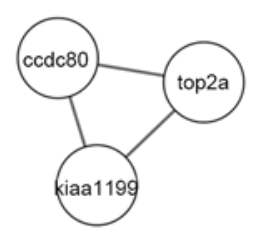

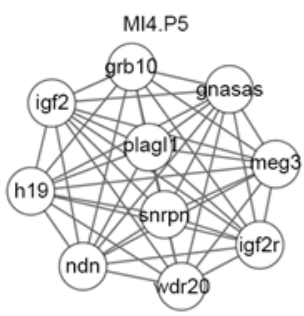

MI76.P103

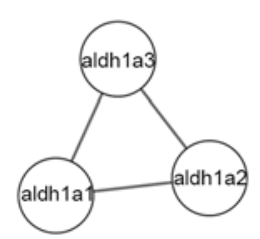

MI86.P97

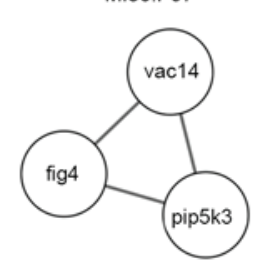

MI38.P55

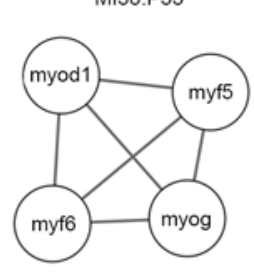

M185.P99

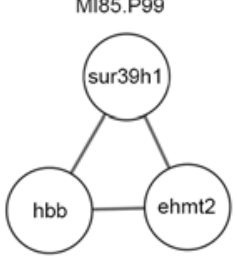

C

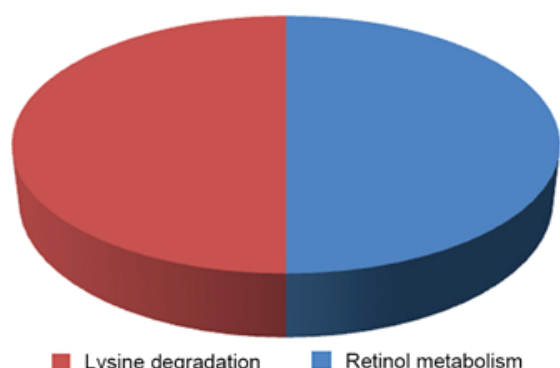

B
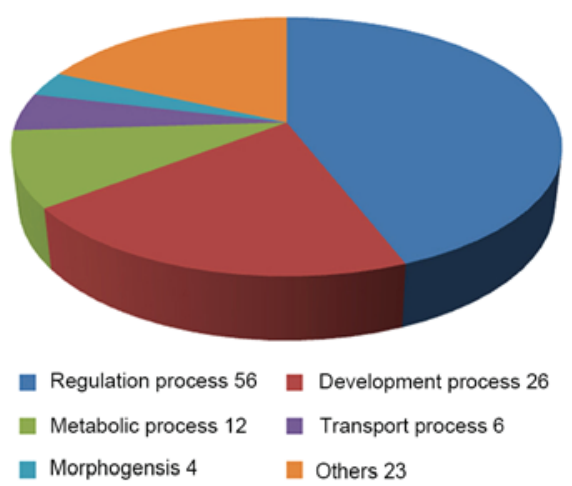

Morphogensis 4 Others 23

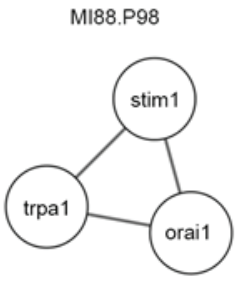

Figure 5. (A) The 9 overlapping modules in the IPAH and PHD networks. (B) The 127 biological processes of the 9 overlapping modules in the IPAH and PHD networks. (C) The 2 pathways of the 9 overlapping modules in the IPAH and PHD networks. CHD, coronary heart disease; IPAH, idiopathic pulmonary arterial hypertension; PHD, pulmonary heart disease.

modules (Fig. 4A), which consisted of a minimum of 3 nodes and 3 edges. The largest one, $\mathrm{M}_{\mathrm{PICl}}$, comprises 26 nodes and 325 edges. The 5 overlapping modules have 176 functional annotations (Fig. 4B) and 5 pathways (Fig. 4C). Regulatory processes, cellular processes and development processes have the most functional annotations. The most significant module, 
A

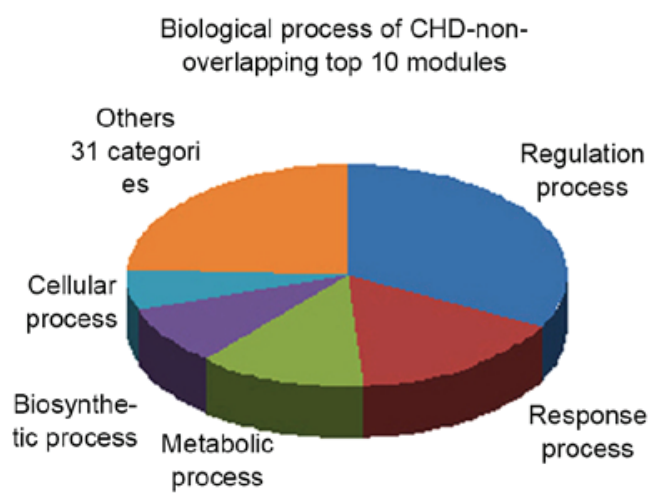

C

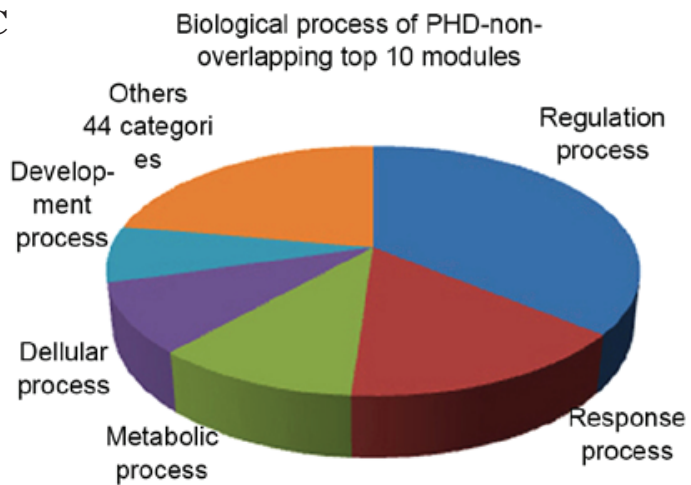

B

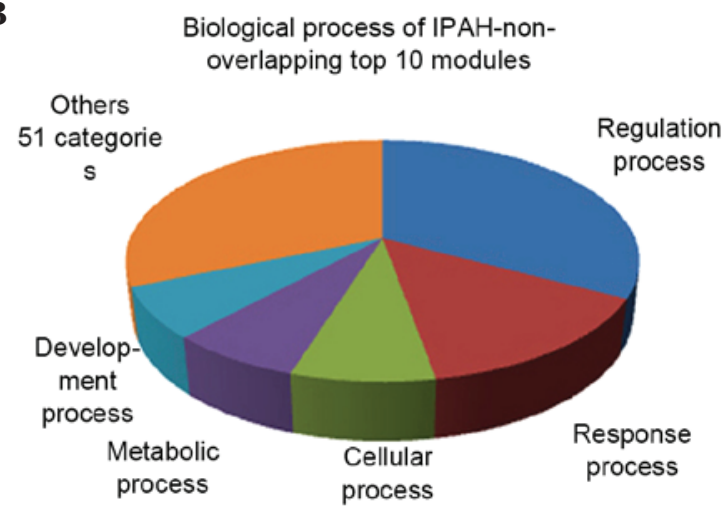

D

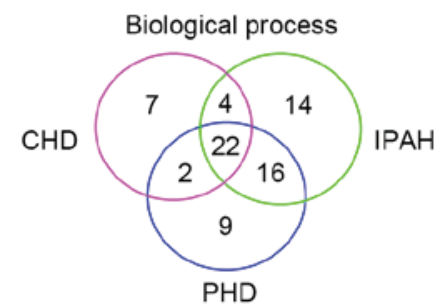

$\mathbf{E}$

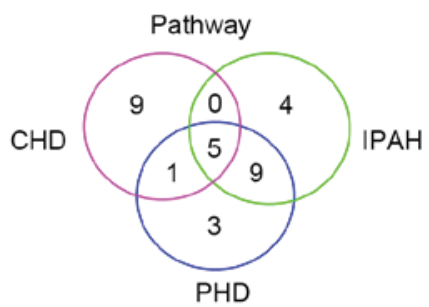

Figure 6. Biological processes of the top 10 non-overlapping modules for (A) CHD, (B) IPAH and (C) PHD. (D) Common biological processes of the top 10 non-overlapping modules. (E) Top 10 non-overlapping modules of the three diseases shared 5 types of common pathways. CHD, coronary heart disease; IPAH, idiopathic pulmonary arterial hypertension; PHD, pulmonary heart disease.

$\mathrm{M}_{\mathrm{P} 1 \mathrm{Cl}}$, is the highest ranked module for PHD and CHD (ranked using the MCODE score) and includes 114 functional annotations, 37 for cellular processes (cell motility, cell migration and other cell processes), and 7 for response processes (for example the response to estrogen stimulus and positive regulation of response to stimuli).

Modules between IPAH and PHD. IPAH and PHD shared 9 other common modules in addition to the three overlapping modules (Fig. 5A). These were the modules that consisted of a minimum of 3 nodes and 3 edges. The most significant module, $\mathrm{M}_{\mathrm{I} 3 \mathrm{P} 3}$, comprises 12 nodes and 66 edges. The 9 overlapping modules have 127 functional annotations (Fig. 5B) and 2 pathways (Fig. 5C). Regulation processes, development processes and metabolic processes have the most functional annotations. The most significant module, $\mathrm{M}_{\mathrm{I} 3 \mathrm{P} 3}$, ranks third in the PHD and IPAH modules (ranked by MCODE score), including 36 functional annotations, including 10 for transcription (for example regulation of transcription and other transcriptional processes), and 9 for metabolic processes (for example the nitrogen compound metabolic process and other metabolic processes).

Modules between IPAH and CHD. IPAH and CHD shared 3 other overlapping modules in addition to the three overlapping modules for IPAH, CHD and PHD (Fig. 3D). The 3 overlapping modules all consisted of 3 nodes and 3 edges, and had 18 functional annotations (Fig. 3E), including the eicosanoid biosynthetic process, the eicosanoid metabolic process, the fatty acid biosynthetic process and the cellular alkene metabolic process. The arachidonic acid metabolism was the only pathway identified (Fig. 3F).

Unique disease modules contribute their own functions and pathways. The top 10 non-overlapping modules of the CHD-associated network (Fig. 6A), the IPAH-associated network (Fig. 6B) and the PHD-associated network (Fig. 6C) shared 22 common biological processes (Fig. 6D), including regulation, response and cellular processes. They shared 5 types of pathways (Fig. 6E), including the metabolic pathway, a cancer-associated pathway and an apoptosis-associated pathway.

\section{Discussion}

The current study identified numerous biological characteristics among IPAH, CHD and PHD. There were 29 overlapping genes that demonstrated genetic similarity between the three diseases. Genes including nitric oxide synthase 3 (NOS3), angiotensin converting enzyme (ACE), ALOX5, VEGFA, apolipoprotein A-I and endoglin have been identified as biomarkers or therapeutic targets for IPAH, CHD and PHD based on direct evidence from the Comparative Toxicogenomics Database (http://ctdbase.org/), which provides manually curated information regarding the association between genes and disease (14). Previous studies have investigated these biological processes, improving the understanding of their roles in the diseases investigated in the current study. 
For example, studies have demonstrated that NOS3 serves a role in cardiovascular pathology and individual responses to antihypertensive drugs $(15,16)$, and that levels of NOS3 vary with increasing CHD and IPAH occurrence $(17,18)$. Additional studies have suggested that ACE may contribute to the development of IPAH and CHD (19,20). Drugs targeted at ACE, such as ACEIs, are used to treat IPAH, PHD and CHD (21-23).

The common modules for IPAH, PHD and CHD shared two pathways: Glycosphingolipid biosynthesis and the arachidonic acid metabolism. Previous studies have indicated that inhibition of glycosphingolipid synthesis induces a marked reduction of plasma cholesterol and inhibits atherosclerosis development in mice, which may alleviate CHD (24-26). The association between glycosphingolipid biosynthesis and IPAH and PHD remains unclear, however targeting glycosphingolipid biosynthesis may be a novel treatment modality for cardiovascular disease. The pathological processes for IPAH, PHD and CHD involve the arachidonic acid metabolism. A previous study indicated that a lower ratio of serum eicosapentaenoic acid to arachidonic acid is associated with a greater risk of cardiovascular disease, particularly CHD (27). Thromboxane A2 (TXA2) is the major arachidonic acid metabolite. It serves a key role in normal physiology and control of vascular tone (28) and is associated with the development of IPAH (29). TXA2 is also closely associated with the occurrence of pulmonary embolism, which leads to PHD $(30,31)$. Biosynthetic processes are the most functional annotations of the three diseases, and they include the leukotriene (LT) biosynthetic process and the lipid biosynthetic process. LTs are lipid mediators that are derived from the 5-lipoxygenase pathway of arachidonic acid metabolism, and which modulate inflammation in pulmonary arterial hypertension (32). LTs serve a pivotal role in the pathogenesis of atherosclerosis, have a close association with CHD (33), and additionally increase oxygen free radicals involved in the development and maintenance of heart failure, which is a typical symptom of PHD (34). Due to the fact that LTs are important in IPAH, CHD and PHD, the use of agents that influence the LT pathway has potential for the use in treatment for IPAH and PHD $(32,34)$. LTs have additionally been identified to be effective in predicting CHD (33). Functional observations including oxidation reduction, the unsaturated fatty acid biosynthetic process, the glycoprotein biosynthetic process and the eicosanoid metabolic process were additionally identified to be associated with IPAH, CHD and PHD in the current study; however, these observations require further investigation and discussion.

A greater number of overlapping functional modules and functional annotations were identified between pairs of the diseases. The most significant CHD and PHD module was $\mathrm{M}_{\mathrm{plcl}}$, and the most important functional annotations of this module are cellular processes and response processes. Regulation of the response to external stimuli and negative regulation of the defense response may be important in regulating the positive and negative feedback loops that maintain cellular status (35). Cell migration, cell motility, regulation of locomotion and positive regulation of locomotion are considered characteristic aspects in CHD (36). Certain treatments influence cell function. Treatments including atorvastatin, which increases the migration of endothelial progenitor cells, can reduce pulmonary arterial pressure in patients with PHD (37).
The most significant IPAH and PHD module is $\mathrm{M}_{\mathrm{I3P} 3}$, and transcription is the major pathway. Regulation of transcription, negative regulation of the RNA metabolic process and positive regulation of transcription from the RNA polymerase II promoter have been identified to participate in regulation of the DNA transcription into messenger RNA by transcription factors. Therapeutic strategies aimed at transcription factors may be a novel treatment technique for IPAH (38).

There are three common IPAH and CHD modules. There are 18 biological processes, some of which are pathways involved in the development of IPAH and CHD, such as the inflammatory response and the response to wounding (39-41). These two diseases are not connected as closely as those described above, potentially due to the fact that the course of IPAH is shorter than that of CHD, and numerous patients are diagnosed with IPAH at a younger age (42). Patients with CHD are predominantly over the age of 40 years (43), and thus there is a lack of overlap for CHD and IPAH.

Medication aimed at common target genes and pathways has been used to treat IPAH and CHD. HMG-coenzyme-A reductase inhibitors (statins) $(44,45)$ and ACEIs $(46,47)$ are used to treat patients with CHD. It has been previously identified that statins reduce pulmonary hypertension in patients with pulmonary heart disease by improving endothelial progenitor cell function (37), and ACEIs are also used to relieve symptoms of IPAH $(48,49)$. The nonselective endothelin (ET) receptor antagonist bosentan, which is recommended as a first-line agent for the treatment of IPAH, improves the hemodynamics and exercise capacity in patients with PHD (50). It has been observed that ET-1 expression is enhanced in human atherosclerotic lesions (51), and ET-1 plasma levels are increased in patients with acute myocardial infarction (a type of CHD) (52). Long-term administration of ET receptor antagonists improves coronary endothelial function in patients with early atherosclerosis (53), and it provides a new approach for prevention and therapy of CHD.

The top 10 non-overlapping IPAH, CHD and PHD modules shared 22 types of similar biological processes, including regulation processes, response processes and cellular processes, which were discussed above and their correlation was presented with the three diseases (35-37). The apoptosis pathway participates in the development of CHD, IPAH and PHD (54-56). Although these top 10 modules are unique, there are also several common types of biological processes and some common pathways, including apoptosis, cell cycle and metabolism. The unique part of the biological processes was also identified to be associated with the disease. Regeneration-associated functions are uncommon in CHD, and intracoronary autologous bone marrow cell transplantation has been reported to result in regeneration of function of human infarcted hearts in patients with CHD (57). The current study demonstrated that there are correlations between IPAH, CHD and PHD, which confirms the necessity and feasibility of the study.

Due to the fact that numerous overlapping genes, biological processes and pathways were identified in the present study, further insight into the pathological mechanisms of IPAH, CHD and PHD was gained, and novel focuses for future research and potential drug targets were identified. The results of the current study suggest that drug indications may be broadened because of these potential targets. 
With the variation present between the large amount of online data available, the results of network analyses may slightly differ. In addition, certain results cannot be fully understood due to the limitations present in the current literature. The current study was based on gene network analysis constructing a system network to provide comprehensive insight into IPAH, PHD and CHD, instead of focusing on a single gene or pathway as previous studies have done.

Overlapping genes and modules in IPAH, PHD and CHD were investigated in the present study. A disease network was constructed to determine the common characteristics between IPAH, PHD and CHD. The three common modules, $\mathrm{M}_{\mathrm{C} 919 \mathrm{P} 13}$, $\mathrm{M}_{\mathrm{C} 1913 \mathrm{P} 25}$ and $\mathrm{M}_{\mathrm{C} 70177 \mathrm{P} 102}$, can be studied together when observing intervention results, and multi-gene alterations in IPAH, PHD and CHD can be studied. In addition, the observations of the current study suggest that drug indications may be broadened due to common targets. For example, medicine aimed at ET to treat IPAH may additionally be used for PHD and CHD.

\section{References}

1. Souza R, Jardim C and Humbert M: Idiopathic pulmonary arterial hypertension. Semin Respir Crit Care Med 34: 560-567, 2013.

2. Guo YH, Su LX, Guo N and Liu CT: Novel therapy for idiopathic pulmonary arterial hypertension: Can hepatocyte growth factor be beneficial? J Geriatr Cardiol 9: 211-212, 2012.

3. Janczura M, Bochenek G, Nowobilski R, Dropinski J, KotulaHorowitz K, Laskowicz B, Stanisz A, Lelakowski J and Domagala T: Correction: The relationship of metabolic syndrome with stress, coronary heart disease and pulmonary function - An occupational cohort-based study. PLoS One 10: e0139408, 2015.

4. Runo JR and Loyd JE: Primary pulmonary hypertension. Lancet 361: 1533-1544, 2003.

5. Patel RS and Ye S: Genetic determinants of coronary heart disease: new discoveries and insights from genome-wide association studies. Heart 97: 1463-1473, 2011.

6. Lewczuk J: Pulmonary heart disease - cardiologist's point of view. Pneumonol Alergol Pol 80: 541-545, 2012 (In Polish).

7. Mikhail G, Chester AH, Gibbs JS, Borland JA, Banner NR and Yacoub $\mathrm{MH}$ : Role of vasoactive mediators in primary and secondary pulmonary hypertension. Am J Cardiol 82: 254-255, 1998.

8. Han MK, McLaughlin VV, Criner GJ and Martinez FJ: Pulmonary diseases and the heart. Circulation 116: 2992-3005, 2007.

9. Fihn SD, Gardin JM, Abrams J, Berra K, Blankenship JC, Dallas AP, Douglas PS, Foody JM, Gerber TC, Hinderliter AL, et al: 2012 ACCF/AHA/ACP/AATS/PCNA/SCAI/STS guideline for the diagnosis and management of patients with stable ischemic heart disease: A report of the American College of Cardiology Foundation/American Heart Association task force on practice guidelines, and the American College of Physicians, American Association for Thoracic Surgery, Preventive Cardiovascular Nurses Association, Society for Cardiovascular Angiography and Interventions, and Society of Thoracic Surgeons. Circulation 126: e354-e471, 2012

10. Csermely P, Korcsmáros T, Kiss HJ, London G and Nussinov R: Structure and dynamics of molecular networks: A novel paradigm of drug discovery: A comprehensive review. Pharmacol Ther 138: 333-408, 2013.

11. Ichihara S, Yamamoto K, Asano H, Nakatochi M, Sukegawa M, Ichihara G, Izawa H, Hirashiki A, Takatsu F, Umeda H, et al: Identification of a glutamic acid repeat polymerphism of ALMS1 as a novel genetic risk marker for early-onset myocardial infarction by genome-wide linkage analysis. Circ Cardiovasc Genet 6: 569-578, 2013.

12. Eaton CB, Gramling R, Parker DR, Roberts MB, Lu B and Ridker PM: Prospective association of vascular endothelial growth factor-A (VEGF-A) with coronary heart disease mortality in southeastern New England. Atherosclerosis 200: 221-227, 2008.

13. Hamosh A, Scott AF, Amberger JS, Bocchini CA, Valle D and McKusick VA: Online mendelian inheritance in Man (OMIM), a knowledgebase of human genes and genetic disorders. Nucleic Acids Res 30: 52-55, 2002
14. Mattingly CJ, Colby GT, Forrest JN and Boyer JL: The Comparative Toxicogenomics Database (CTD). Environ Health Perspect 111: 793-795, 2003.

15. van der Wal AC: Coronary artery pathology. Heart 93: 1484-1489, 2007.

16. Cooke GE, Doshi A and Binkley PF: Endothelial nitric oxide synthase gene: Prospects for treatment of heart disease. Pharmacogenomics 8: 1723-1734, 2007.

17. Colombo MG, Paradossi U, Andreassi MG, Botto N, Manfredi S, Masetti S, Biagini A and Clerico A: Endothelial nitric oxide synthase gene polymorphisms and risk of coronary artery disease. Clin Chem 49: 389-395, 2003.

18. Aytekin M, Aulak KS, Haserodt S, Chakravarti R, Cody J, Minai OA and Dweik RA: Abnormal platelet aggregation in idiopathic pulmonary arterial hypertension: Role of nitric oxide. Am J Physiol Lung Cell Mol Physiol 302: L512-L520, 2012.

19. Orte C, Polak JM, Haworth SG, Yacoub MH and Morrell NW: Expression of pulmonary vascular angiotensin-converting enzyme in primary and secondary plexiform pulmonary hypertension. J Pathol 192: 379-384, 2000.

20. Mattu RK, Needham EW, Galton DJ, Frangos E, Clark AJ and Caulfield M: A DNA Variant at the angiotensin-converting enzyme gene locus associates with coronary artery disease in the caerphilly heart study. Circulation 91: 270-274, 1995.

21. KannoS,Wu YJ,LeePC,BilliarTR andHoC:Angiotensin-converting enzyme inhibitor preserves p21 and endothelial nitric oxide synthase expression in monocrotaline-induced pulmonary arterial hypertension in rats. Circulation 104: 945-950, 2001.

22. McCullough PA: Chronic kidney disease: Tipping the scale to the benefit of angiotensin-converting enzyme inhibitors in patients with coronary artery disease. Circulation 114: 6-7, 2006.

23. Zisman LS, Asano K, Dutcher DL, Ferdensi A, Robertson AD, Jenkin M, Bush EW, Bohlmeyer T, Perryman MB and Bristow MR: Differential regulation of cardiac angiotensin converting enzyme binding sites and AT1 receptor density in the failing human heart. Circulation 98: 1735-1741, 1998.

24. Bietrix F, Lombardo E, van Roomen CP, Ottenhoff R, Vos M, Rensen PC, Verhoeven AJ, Aerts JM and Groen AK: Inhibition of glycosphingolipid synthesis induces a profound reduction of plasma cholesterol and inhibits atherosclerosis development in APOE*3 Leiden and low-density lipoprotein receptor-/-mice. Arterioscler Thromb Vasc Biol 30: 931-937, 2010.

25. Peters SA, Singhateh Y, Mackay D, Huxley RR and Woodward M: Total cholesterol as a risk factor for coronary heart disease and stroke in women compared with men: A systematic review and meta-analysis. Atherosclerosis 248: 123-131, 2016.

26. Chapman MJ: Therapeutic elevation of HDL-cholesterol to prevent atherosclerosis and coronary heart disease. Pharmacol Ther 111: 893-908, 2006.

27. Ninomiya T, Nagata M, Hata J, Hirakawa Y, Ozawa M, Yoshida D, Ohara T, Kishimoto H, Mukai N, Fukuhara M, et al: Association between ratio of serum eicosapentaenoic acid to arachidonic acid and risk of cardiovascular disease: The Hisayama Study. Atherosclerosis 231: 261-267, 2013

28. Ball SK, Field MC and Tippins JR: Regulation of thromboxane receptor signaling at multiple levels by oxidative stress-induced stabilization, relocation and enhanced responsiveness. PLoS One 5: e12798, 2010.

29. Hirenallur-S DK, Detweiler ND, Haworth ST, Leming JT, Gordon JB and Rusch NJ: Furegrelate, a thromboxane synthase inhibitor, blunts the development of pulmonary arterial hypertension in neonatal piglets. Pulm Circ 2: 193-200, 2012.

30. Sobieszczyk P, Fishbein MC and Goldhaber SZ: Acute pulmonary embolism: Don't ignore the platelet. Circulation 106: 1748-1749, 2002.

31. Goldhaber SZ and Elliott CG: Acute pulmonary embolism: Part I epidemiology, pathophysiology, and diagnosis. Circulation 108: 2726-2729, 2003

32. Tian W, Jiang X, Sung YK, Qian J, Yuan K and Nicolls MR: Leukotrienes in pulmonary arterial hypertension. Immunol Res 58: 387-393, 2014.

33. Nair J, Shanker J, Jambunathan S, Arvind P and Kakkar VV: Expression analysis of leukotriene-inflammatory gene interaction network in patients with coronary artery disease. J Atheroscler Thromb 21: 329-345, 2014.

34. Prasad K and Kalra J: Oxygen free radicals and heart failure. Angiology 39: 417-420, 1988 .

35. Kim D, Kwon YK and Cho KH: Coupled positive and negative feedback circuits form an essential building block of cellular signaling pathways. Bioessays 29: 85-90, 2007. 
36. Karastergiou K, Evans I, Ogston N, Miheisi N, Nair D, Kaski JC, Jahangiri $\mathrm{M}$ and Mohamed-Ali V: Epicardial adipokines in obesity and coronary artery disease induce atherogenic changes in monocytes and endothelial cells. Arterioscler Thromb Vasc Biol 30: 1340-1346, 2010

37. Liu HF, Qi XW, Ma LL, Yao DK and Wang L: Atorvastatin improves endothelial progenitor cell function and reduces pulmonary hypertension in patients with chronic pulmonary heart disease. Exp Clin Cardiol 18: e40-e43, 2013.

38. Crosswhite P and Sun Z: Molecular mechanisms of pulmonary arterial remodeling. Mol Med 20: 191-201, 2014.

39. Hamidi SA, Prabhakar S and Said SI: Enhancement of pulmonary vascular remodelling and inflammatory genes with VIP gene deletion. Eur Respir J 31: 135-139, 2008.

40. Heusch G, Schulz R and Erbel R: Inflammatory markers in coronary heart disease: Coronary vascular versus myocardial origin? Circulation 108: e4, 2003.

41. de Nigris F, Youssef T, Ciafré S, Franconi F, Anania V, Condorelli G, Palinski W and Napoli C: Evidence for oxidative activation of c-Myc-Dependent nuclear signaling in human coronary smooth muscle cells and in early lesions of watanabe heritable hyperlipidemic rabbits: Protective effects of vitamin E. Circulation 102: 2111-2117, 2000.

42. Fraisse A, Jais X, Schleich JM, di Filippo S, Maragnès P, Beghetti M Gressin V, Voisin M, Dauphin C, Clerson P, et al: Characteristics and prospective 2-year follow-up of children with pulmonary arterial hypertension in France. Arch Cardiovasc Dis 103: 66-74, 2010.

43. Page IH, Berrettoni JN, Butkus A and Sones FM Jr: Prediction of coronary heart disease based on clinical suspicion, age, total cholesterol, and triglyceride. Circulation 42: 625-645, 1970.

44. Vaughan CJ, Gotto AM Jr and Basson CT: The evolving role of statins in the management of atherosclerosis. J Am Coll Cardiol 35: 1-10, 2000.

45. Maron DJ, Fazio S and Linton MF: Current perspectives on statins. Circulation 101: 207-213, 2000.

46. Yusuf S, Sleight P, Pogue J, Bosch J, Davies R and Dagenais G: Effects of an angiotensin converting-enzyme inhibitor, ramipril, on cardiovascular events in high-risk patients. The heart outcomes prevention evaluation study investigators. N Engl J Med 342: $145-153,2000$
47. Yusuf S, Sleight P, Pogue J, Bosch J, Davies R and Dagenais G: Cardiac rehabilitation and secondary prevention of coronary heart disease. Circulation 111: 369-376, 2005.

48. Jeffery TK and Wanstall JC: Pulmonary vascular remodeling: A target for therapeutic intervention in pulmonary hypertension. Pharmacol Ther 92: 1-20, 2001.

49. Budhiraja R, Tuder RM and Hassoun PM: Endothelial dysfunction in pulmonary hypertension. Circulation 109: 159-165, 2004

50. Rich S and McLaughlin VV: Endothelin receptor blockers in cardiovascular disease. Circulation 108: 2184-2190, 2003.

51. Minamino T, Kurihara H, Takahashi M, Shimada K, Maemura K, Oda H, Ishikawa T, Uchiyama T, Tanzawa K and Yazaki Y: Endothelin-converting enzyme expression in the rat vascular injury model and human coronary atherosclerosis. Circulation 95: 221-230, 1997.

52. Stewart DJ, Kubac G, Costello KB and Cernacek P: Increased plasma endothelin-1 in the early hours of acute myocardial infarction. J Am Coll Cardiol 18: 38-43, 1991.

53. Reriani M, Raichlin E, Prasad A, Mathew V, Pumper GM, Nelson RE, Lennon R, Rihal C, Lerman LO and Lerman A: Long-term administration of endothelin receptor antagonist improves coronary endothelial function in patients with early atherosclerosis. Circulation 122: 958-966, 2010.

54. Blanco-Colio LM: TWEAK/Fn14 Axis: A promising target for the treatment of cardiovascular diseases. Front Immunol 5: 3, 2014.

55. Zhang S, Fantozzi I, Tigno DD, Yi ES, Platoshyn O, Thistlethwaite PA, Kriett JM, Yung G, Rubin LJ and Yuan JX: Bone morphogenetic proteins induce apoptosis in human pulmonary vascular smooth muscle cells. Am J Physiol Lung Cell Mol Physiol 285: L740-L754, 2003.

56. Merklinger SL, Jones PL, Martinez EC and Rabinovitch M: Epidermal growth factor receptor blockade mediates smooth muscle cell apoptosis and improves survival in rats with pulmonary hypertension. Circulation 112: 423-431, 2005.

57. Strauer BE, Brehm M, Zeus T, Bartsch T, Schannwell C, Antke C, Sorg RV, Kögler G, Wernet P, Müller HW and Köstering M: Regeneration of human infarcted heart muscle by intracoronary autologous bone marrow cell transplantation in chronic coronary heart disease: The IACT-study. J Am Coll Cardiol 46: 1651-1658, 2005 . 